\title{
Wideband amplification of transient signals for application in pulse compression
}

\author{
A.R. Young, C.G. Whyte, C.W. Robertson, P. Maclnnes, \\ A.D.R. Phelps, A.W. Cross, L. Zhang, C.R. Donaldson, \\ K. Matheson and K. Ronald
}

SUPA and Department of Physics, University of Strathclyde, Glasgow, G4 ONG, UK

Helically corrugated waveguide can be used as a dispersive medium to compress long duration chirping signals into higher power shorter duration microwave pulses1,2,3. It has the advantage over smooth bore circular waveguide in that its dispersion can be shaped to have a large change in group velocity over a relatively small frequency range, but still be far from 'cut-off' where there is a high risk of reflection.

The use of such a waveguide as a pulse compressor has been demonstrated using either a travelling wave tube, TWT1 or a relativistic linear-beam Backward Wave Oscillator4, BWO. Although a TWT provides relatively low power, its ability to follow a low power drive signal gives flexibility in the shaping of the input. A BWO can supply a high power input pulse, but its tuning can be hard to map precisely to the compressor dispersion.

A helical waveguide gyro Travelling Wave Amplifier5,6, gyro-TWA, is an attractive final stage in an amplifier chain for driving a dispersive pulse compressor. This has the flexibility of a conventional TWT in allowing precise control over the shape and sweep of the input pulse, while supplying significantly more power. A computer model of a gyro-TWA is being used to predict its operation with steady-state single frequency signals and spread spectrum and chirp pulses over a frequency range suitable for such a pulse compressor. Output from the amplifier model can be used in the simulation of the pulse compressor to predict the optimum input chirp, with the objective of confirming this experimentally.

Support from the US AFOSR and prior support from the UK EPSRC, STFC and dstl are gratefully acknowledged.

1. Samsonov S.V., Phelps A.D.R., et al., Phys. Rev. Lett., 92, 118301, 2004

2. Burt G., Samsonov S.V., et al., Phys. Rev. E, 70, Art. No. 046402, 2004.

3. Zhang L., Mishakin S.V., et al., IEEE Trans. Microw. Theory Techn., 63, pp. 1090-1096, 2015.

4. Bratman V.L., Denisov G.G., et al., Phys. Plasmas, 17, 110703, 2010.

5. Bratman V.L., Cross A.W., et al., Phys. Rev. Lett., 84, pp. 2746-2749, 2000.

6. Whyte C.G., Ronald K., Young A.R., et al., IEEE Trans. Plasma Sci., 40, pp. 1303-1310, 2012.

This is a peer-reviewed, accepted author manuscript of the following article: Young, A., Whyte, C., Robertson, C., Maclnnes, P., Phelps, A., Cross, A., Zhang, L., Donaldson, C., Matheson, K., \& Ronald, K. (2021). Wideband amplification of transient signals for application in pulse compression. In 2021 IEEE International Conference on Plasma Science (ICOPS) 2021 (IEEE International Conference on Plasma Science (ICOPS)). IEEE. https://doi.org/10.1109/ ICOPS36761.2021.9588422 